JOURNAL OF

APPLIED

CRYSTALLOGRAPHY

ISSN 1600-5767

\section{Introduction to Scanning Tunneling Microscopy. Second Edition. By C. Julian Chen. Oxford University Press, 2015. Pp. 488. Price GBP 39.99 (paperback). ISBN 9780198754756.}

\author{
J. A. A. W. Elemans*
}

Institute for Molecules and Materials (IMM), Radboud University, Heijendaalseweg 135, 6525 AJ Nijmegen, The Netherlands. *Correspondence e-mail: j.elemans@science.ru.nl

In the past three decades the field of scanning probe microscopy (SPM) research has rapidly expanded. Hand in hand with the development of a new generation of microscopes that can provide the highest resolution possible, the ability to characterize surfaces down to the molecular or atomic level by means of direct visualization has become an essential and highly appealing aspect of nanoscience. Initially specialist tools, scanning tunneling microscopes and atomic force microscopes have found their way into a wide range of laboratories, spanning disciplines from elementary physics to biomedical research. This also means that scientists with significantly varying backgrounds need to learn how to work with such microscopes. The book Introduction to Scanning Tunneling Microscopy by C. Julian Chen serves as an excellent starting point to familiarize newcomers with the field, and at the same time provides an in-depth account of theoretical and practical aspects of SPM for the more experienced user. In my personal experience it is also very useful as a textbook for teaching single-molecule studies, at both the beginners' and the advanced level.

The book opens with an attractive gallery of photographs that illustrate the development of scanning tunneling microscopy (STM) and some typical microscope images of surfaces at the atomic scale. Next, a compact overview chapter introduces the SPM techniques, their basic principles, scope and shortcomings, and some applications. This part is written in a comprehensible style which makes it highly suitable for the unexperienced SPM experimentalist. Then the book is divided into two major sections that discuss the concepts of SPM in depth: Principles, which focuses on the fundamental theory and imaging aspects of STM and atomic force microscopy (AFM), and Instrumentation, which addresses the construction and practical use of scanning probe microscopes.

The first section of the book deals with the fundamental physics behind STM and AFM. It starts with a description of the tunneling phenomenon between a tip and a sample, using a modified version of Bardeen's perturbation theory. Taking this theory as a guide, atomic forces and atomic scale tunneling are treated in detail, leading to the description of the local density of states, which is the key to interpreting STM images. Then the imaging mechanisms of STM are described with the help of the TersoffHamann theory. The first section is concluded by a chapter dealing with imaging surfaces at the atomic scale, and nano-mechanical effects that occur between tip and sample.

The second section of the book elaborates on the practical aspects of SPM techniques. The heart of the scanning tunneling microscope is the piezoelectric scanning device, which enables tip displacements over subatomic distances in the $\mathbf{x}, \mathbf{y}$ and $\mathbf{z}$ directions. The piezoelectric effect is described, as well as the type of materials and scanners employed in tunneling microscopes. A part dedicated to the essential vibration isolation of the microscopes is followed by a description of electronic data collection and feedback control. Different design types of scanning tunneling microscopes are presented, and a chapter is devoted to ex situ tip construction and in situ tip treatment methods. Towards further characterization of surface structures, scanning tunneling spectroscopy is introduced. A final chapter dealing with AFM provides some extra information about the use of a merely complementary scanning probe technique that has nowadays become a standard tool in many chemical, biological and biomedical laboratories. 
To give a complete picture of a book also its shortcomings should be discussed, but in this case they are not easy to find. As the focus is really on the fundamentals of the technique, one should not expect extensive descriptions of all types of SPM modes that have been developed over the years, or an elaborate discussion of nanoscale surface phenomena. A small shortcoming in the overview chapter is that for SPM at liquidsolid interfaces only aqueous solvents are mentioned. In the almost 25 years since the first edition of the book a large STM community has developed in which functional molecular networks are self-assembled at interfaces of surfaces and nonvolatile organic liquids, employing very simple setups that do not require multi-electrode designs. The resolution achieved in such relatively 'dirty' environments nowadays challenges results obtained in ultra-clean ultra-high-vacuum systems. That said, plenty of dedicated reviews that specifically cover the above topics have appeared over the years in the scientific literature. As a guide to the reader, several of these reviews are already mentioned by the author in the preface to the book.

In conclusion, Introduction to Scanning Tunneling Microscopy is an excellent book that can serve as a standard introduction for everyone that starts working with scanning probe microscopes, and a useful reference work for those more advanced in the field. Since their invention in the early 1980s, scanning tunneling microscopes and atomic force microscopes have been under continuous development and their applications and abundance in nanoscience have expanded dramatically. For everyone working in this field, the book by C. Julian Chen remains an indispensable basis, as well as an essential prerequisite for understanding the more specialized books and reviews on scanning probe microscopy. 